\title{
Diseño de una experiencia de Flip-Teaching para la asignatura Deontología y Profesionalismo a impartir en la Escuela Técnica Superior de Ingeniera Informática de la UPV
}

\author{
Juan Vte. Oltra Gutiérrez
}

aUniversitat Politècnica de Valencia, jvoltra@omp.upv.es.

\begin{abstract}
In this paper, we will see the basic lines that make up the design of DyP, subject of the UPV-ETSINF in flip-teaching format.

First, we will see a brief description of the environment: DyP in the degree, and how are the students: their roots, knowledge and experiences.

Secondly we present DyP, focusing on their skills and content, and their classification.

Then we enumerate the different tools that the university has made available to us to meet the challenge and, in time, will evaluate the possibilities were shuffled to use the proposed educational purposes.

With this, we will be ready to present the various strategies that will shape the design of teaching, classifying the materials available and predictable, and the various means of communication to be used with students.

This would put us in a position to present a picture of planned evaluations to verify that the powers are reached.

We would end with brief conclusions and estimates and forecast improvement, overlooking the second edition in the 2015-2016 year.
\end{abstract}

Keywords:Flip Teaching; evaluation; Tools to support teaching

\section{Resumen}

En el presente trabajo, se exponen las líneas básicas que conforman el diseño de la asignatura DyP, a impartir en formato flip-teaching en la ETSINF de la UPV.

En primer lugar, haremos una breve descripción del entorno: la asignatura dentro de la titulación, y cómo es la población que va a cursar la materia, 
Diseño de una experiencia de Flip-Teaching para la asignatura Deontología y Profesionalismo a impartir en la Escuela Técnica Superior de Ingeniera Informática de la UPV

esto es, la procedencia del alumno y sus conocimientos y experiencias previas.

En segundo lugar presentamos la materia, centrándonos en sus competencias y contenidos, y en la clasificación de éstos.

A continuación, enumeraremos las distintas herramientas que la universidad ha puesto a nuestro alcance para poder afrontar el reto y, al tiempo, evaluaremos las posibilidades que se barajaron para poder usarlas con los fines docentes propuestos.

Con ello, estaremos en disposición de presentar las distintas estrategias que conformarán el diseño de la docencia, clasificando los materiales disponibles y previsibles, así como las distintas vías de comunicación que se emplearán con el alumnado.

Esto no pondría en condiciones de presentar un cuadro de evaluaciones previstas que permitan verificar que las competencias se alcanzan.

Finalizaríamos con unas breves conclusiones y previsiones de mejora y previsión, con vista a la segunda edición de la misma en el curso 2015-2016.

Palabras clave:Flip Teaching; Evaluación; Herramientas de apoyo a la docencia

\section{Introducción}

La presente experiencia nace fruto de una iniciativa del Vicerrectorado de las Tecnologías de la Información y las Comunicaciones, quien impulsa con un horizonte definido a priori en dos cursos académicos, un grupo de cada una de las asignaturas de las titulaciones de Administración de Empresas e Informática en segundo curso de grado, como Flip-Teaching (término que en el ámbito docente está ya lo suficientemente extendido como para permitirnos obviar una definición formal)

La asignatura objeto del presente trabajo, se trata de Deontología y Profesionalismo, obligatoria que se imparte durante el segundo semestre del curso segundo de la Escuela Superior de Ingeniería Informática.

La asignatura en sí tiene unas características que la hacen particularmente distinta al resto como podemos apreciar simplemente dando un vistazo a los nombres del resto de asignaturas que comparten semestre: Estructura de computadores, Redes de computadores, Concurrencia y sistemas distribuidos, Estructuras de datos y algoritmos e Interfaces persona 
computador. Ésto nos permite con una rápida apreciación intuitiva, que resulta confirmada por la realidad, bosquejar la idea de que el alumno ve en el resto de sus asignaturas una practicidad inmediata, mientras que en ésta, donde se les habla de las buenas prácticas y la deontología profesional, además de darles una visión del marco legal en el que se van a mover, resultará a priori para su percepcion algo más apartado de su día a día, lo que implica un mayor esfuerzo del docente para lograr su implicación.

Por concretar un poco más, se intenta acercar al alumno a la legislación más importante que afecta a su desarrollo profesional (Protección de datos, Propiedad intelectual -tipos de licencias-, Código penal, LSSI y otras). A los aspectos deontológicos y éticos de la profesión y el estudio de los códigos éticos más significativos, se añade el análisis del profesionalismo y competencias en el ámbito de las TI y una visión del asociacionismo y colegios profesionales.

Los contenidos están divididos en tres categorías, tras un tema introductorio: Profesionalismo Informático, donde ven conceptos básicos de profesionalismo e historia de la profesión informática, y relacionados con el profesional en la organización y fuera de ella; Aspectos Legales en la Informática, donde partiendo de conceptos básicos y marco legal de la actividad del profesional informático, se pone acento en protección de datos, propiedad intelectual y pruebas peritciales informáticas; y una tercera parte, Deontología Informática, donde partiendo de conceptos básicos se llega a las particularidades de la deontología informática: lo qué nos diferencia de otras profesiones

Temas, como hemos visto, con suficiente disimilitud con el resto de las asignaturas como para que resulte al docente un reto el lograr su homologación con ellas en el imaginario colectivo del alumnado. En la otra parte de la balanza, tenemos el hecho de que se trate de una población con no solo altos conocimientos, sino también fuerte interés en las TIC, lo que nos facilita el uso de recursos que en otras titulaciones no tendrían un éxito claro, más allá del mero visionado de vídeos.

\section{Objetivos}

Se pretende efectuar una revisión de las distintas herramientas disponibles, facilitadas por la universidad o de uso gratuito.

Con las herramientas clasificadas, se presentan distintas estrategias que, haciendo uso de ellas, conformarán el diseño de las actividades docentes.

Éstas actividades conllevan una serie de evaluaciones que permitirán comprobar el alcance de las mismas en la formación de los alumnos. 
Diseño de una experiencia de Flip-Teaching para la asignatura Deontología y Profesionalismo a impartir en la Escuela Técnica Superior de Ingeniera Informática de la UPV

\section{Desarrollo de la innovación}

\section{Herramientas disponibles. Posibilidades de las mismas}

En ésta experiencia flip teaching tratamos de combinar las mejores prácticas de la educación tradicional y los enfoques online, conectándolos para orientar el aprendizaje integrado de cada estudiante. Consideramos, siguiendo a (Vasileva-Stojanovska, 2015) las necesidades de aprendizaje de los estudiantes actuales consumidores masivos de pantallas y no tanto de papel. Por una parte, tratamos de proporcionarles un ambiente adaptativo a las necesidades del estudiante, para intentar alcanzar un rendimiento óptimo. Al tiempo, con una cuidada planificación preparamos su trabajo colaborativo en ciertas tareas, guiándolos por un camino con distintas entregas de trabajos con entregas síncronas y asíncronas de material.

Usamos material que nos permita sustituir la clase directa, de forma que al favorecer que los alumnos la puedan seguir en casa, trasladamos ese tiempo de trabajo allí, liberandonos el tiempo de aula para esas actividades de refuerzo que asumimos deben realizar por su cuenta y que cuya realización no siempre podemos constatar. Además, como nos indica (Sams, 2013) podemos usar ese tiempo para ayudar a los estudiantes que no llegan, con material de apoyo complementario, y lanzar desafíos a aquellos que ya han dominado el contenido, facilitándoles otro tipo de material que de una forma un tanto coloquial, bautizamos con la etiqueta "Para saber más"

Por otra parte, siguiendo el ejemplo de (Roshan, 2011) tratamos de romper otra atadura: la que los liga a un ordenador, sea portatil o de sobremesa. Para ello preparamos material que puede ser consumido también desde un smartphone o una tableta, y así arañaremos tiempo de una mina que suele estar sin explotar: el que habitualmente se pierde en el metro, el autobús... buscando la conversión de los transportes públicos en pequeñas aulas portátiles.

La diferencia fundamental que existe, y provocamos, con los materiales clásicos está en su adaptabilidad. Un estudiante puede volver a recibir una clase, como repaso antes del examen, escuchar de nuevo aquellos conceptos que le queden confusos y, no lo perdamos de vista, todo a su propio ritmo, pues no hay dos alumnos iguales, y el tiempo parecer ir a distinta velocidad en unos casos u otros. Recuperar clases perdidas por enfermedad se hace más fácil y, algo casi imposible, el lograr la concentración en el aula para asimilar conceptos complejos, resuta fácil al poder trasladar el aula su propia casa. (Roshan, 2011)

A la hora de seleccionar las herramientas a emplear, el punto de partida necesario es revisar que herramientas pone a nuestra disposición la Universidad Politécnica de Valencia. Centrándonos en laplataforma Poliformat (basada en Sakai), con sus muchas posibilidades, 
algunas elementales y otras de mayor complejidad, como colgar recursos de distintos tipos, almacenar enlaces, uso de foros, exámenes online, cuestionarios, programación de tareas, uso de chat y correo interno... Conviene destacar desde éste primer momento a una de éstas herramientas en concreto, Contenidos, con la que pudimos elaborar guías que ayuden a los alumnos a descubrir los materiales y a usar el resto de herramientas y funcionalidades de poliformat en el orden adecuado y en el plazo correspondiente.

Añadamos a esto la posibilidad de generar contenidos de calidad, gracias a los estudios de grabación de la universidad que favorecen la creación de vídeos docentes (llamados polimedias), de unos 10 minutos de duración.

Para cubrir la totalidad de la asignatura, se usan una gran diversidad de elementos continentes digitales distintos:

- Ficheros con formato pdf:

o Apuntes de la asignatura, en su versión clásica. Para su estudio reposado, mediante su impresión, envío a reprografía o uso de una pantalla. Aunque de una u otra manera sus contenidos resulten redundantes al encontrarse también repartidos en el resto de contenidos, siempre son un elemento de interés para el alumno.

o Guiones de trabajo para casos de aula o prácticas de laboratorio. Instrucciones precisas para trabajar en el aula como seminario, con adaptaciones de casos reales, o para guía de trabajo durante las prácticas.

o Artículos, documentos de trabajo (normas, códigos) o libros con licencia creative commons, sirven para actividades de refuerzo, o para ayuda al alumno inquieto que quiere "saber más". Siempre identificables como tales.

- Videos:

o Capturas de pantalla (screen cast) y los citados polimedias, vídeos con contenido docente. La creación de éstos y otros materiales con distinto software e instalaciones de apoyo, quedan fuera del ámbito de la presente comunicación, pero resulta conveniente siquiera dejar la idea de que, al margen de los medios que proporciona la universidad, existe una gran cantidad de software de uno libre, con licencias freeware, creative commons, etc., muy útil para tal fin.

o Documentales, con contenido de interés, con licencia creative commons o abiertos en la plataforma de su propietario. Otros vídeos docentes, en plataformas como youtube. Sirven de apoyo a los casos planteados, o para ampliación de conocimientos.

- Audio

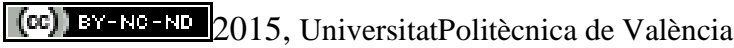

Congreso In-Red (2015) 
Diseño de una experiencia de Flip-Teaching para la asignatura Deontología y Profesionalismo a impartir en la Escuela Técnica Superior de Ingeniera Informática de la UPV

o Ficheros en formato mp3, con resúmenes, definiciones o fragmentos muy breves de teoría, preparados para apoyar actividades de repaso, con plena movilidad del estudiante, que no dependerá así de una pantalla.

Éstos elementos por si sólos no sirven para nada. Es preciso introducirlos en el proceso de comunicación con los alumnos, conduciéndolos en el orden adecuado y estableciendo mecanismos de retorno no solo para evaluar su impacto en ellos, sino para calificarlos. Hablaremos de su ciclo de vida desde el punto de vista del docente en el apartado siguiente.

La herramienta principal que se usa para priorizarlos, darles orden, y que de paso sirve de argamasa para que esos ladrillos se mantengan unidos, conduciendo con textos breves de unos a otros e introduciéndolos, es "Contenidos", de poliformat. "Contenidos" es un simple editor html donde podemos colocar texto, imágenes y, lo más importante, enlaces a los elementos que citábamos antes, permitiendo dar paso a sencillos tests de comprensión sin ue el alumno abandone su navegación por los mismos, que se convierte en algo intuitivo.

Una vez los alumnos disponen del material necesario, pautado en tiempos, se hace necesario que el profesor no quede a ciegas, sin saber si van digiriendo la información en su poder, más allá de la clásica evaluación mediante un examen en papel, que reviste el peligro de llegar tarde para enmendar problemas que puedan ir surgiendo.

Para ello, se emplean distintas herramientas presentes que superan al examen permitiendo un uso asíncrono de las mismas, en el que docente y discente no tienen porqué coincidir. Algunas, como los "Foros", requieren de un seguimiento costoso en tiempo para el profesor. Otros, como los "Exámenes" online o las "Encuestas" que proporciona poliformat, permiten distintos grados de automatización, según sea la construcción de las preguntas empleadas. Todo ello sin perder de vista que para las entregas de pequeños trabajos o resultados de prácticas, mediante la programación de "Tareas" que nos permite la plataforma, no solo el camino del alumno queda balizado, sino que la corrección del profesor, se agiliza mucho, más si consideramos el nuevo sistema antiplagio, recientemente incorporado.

\section{Estrategias docentes}

Con la modalidad tradicional, donde prima la clase magistral en el aula, el margen de maniobra se reduce a probar distintas alternativas de evaluación: pequeños trabajos, microexámenes en aula tras revisión de casos... tras una lenta evolución, los pesos en los mecanismos de evaluación de la asignatura quedaron repartidos como $25 \%$ por trabajos de prácticas, 35\% por exámenes (distribuido en dos parciales) y 40\% por casos (a razón de cuatro casos en sesiones de seminario).

(c) ) EY-NC-ND 2015, UniversitatPolitècnica de València

Congreso IN-RED (2015) 
Al trasladar ese tiempo de teoría fuera del aula, se hace imprescindible una adaptación para los alumnos que cursan la materia en el grupo FLIP. Adaptación encaminada a ir verificando que el alumno, está asimilando los conceptos de la manera esperada, para poder apoyarle en caso contrario.

Introducir cambios implica un desafío queenfrentamos a través de la continua revisión de la planificación (Mowafy, 2013). Podemos verlo gráficamente en la figura siguiente:

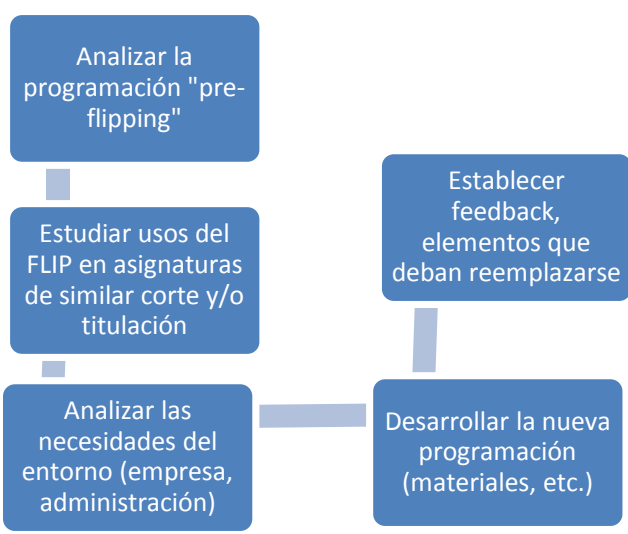

Fig. 1 Cambios en la programación. Elaboración propia, basada en Mowafy

El eje del presente trabajo, resulta obvio, está en la cuarta burbuja, desarrollar la nueva programación.

Tras el preceptivo repaso de bibliografía, desde clásicos ((Bloom, 1981) a ideas más innovadoras (Domingo, 2015), y con las consultas de rigor a compañeros con experiencia en éstas lides, se ha optado por transformar la parte de lo que podemos llamar teoría pura, en material de autoconsumo por los alumnos (vídeos y audios docentes en su mayor parte, pero no solo eso, según la relación ut supra). El trabajo de seminario se ha multiplicado, para ocupar el tiempo de aula dejado libre, manteniendo en parte la estructura ya empleada antes de la experiencia flip (debate en aula y realización de una pequeña prueba) pero ampliando el número de casos vistos y el tiempo dedicado a ellos, todo ello complementado con nuevo material para el trabajo y refuerzo en casa, creado ex profeso, y el uso de foros y exámenes online para poder sacar del aula también parte de estas tareas.

Tenemos como punto de partida, unas competencias que los alumnos deben alcanzar, idénticas para cualquier grupo de la asignatura y presentes en la guía docente. Unido a esto, además, hay un temario muy claro. Todo esto hay que adaptarlo en el tiempo y en el espacio. Para conseguirlo, la secuencia para el docente, tiene un esquema muy simple: en 
Diseño de una experiencia de Flip-Teaching para la asignatura Deontología y Profesionalismo a impartir en la Escuela Técnica Superior de Ingeniera Informática de la UPV

primer lugar, generamos una distribución temporal de la asignatura, estudiando consideraciones como periodos vacacionales (que no deben “cortar” temas), trabajo presencial y no presencial del alumno. Con esta plantilla, preparamos los materiales y las evaluacioens sobre los mismos, secuenciados en el tiempo.

Sobre la creación de materiales, en los casos de uso directo en aula, poco hay que decir. El cambio viene cuando van a ser sacados de ella. Sean documentos de texto, audios y vídeos propios, polimedias y screencast, seguimos para ello los consejos de (Sams, 2013): planificar la clase, grabar, editar (algo no disponible para los polimedias, que obligan a la repetición de la grabación) y publicar.

La primera fase, la planificación, que incluye no solo el calendario sino la modalidad, presencial o no, en que los objetivos se van a cubir, es la parte más compleja. En esa fase como dice (Spencer, 2011) nos preguntamos ¿que queremos que dominen los alumnos al final? Todo es susceptible de ser enlatado, de una u otra manera. Lo importante no es la herramienta, sino el éxito logrado. Así, reservamos los aspectos más complejos para las sesiones presenciales. En cualquier caso, buscamos el pensamiento crítico, el trabajo colaborativo (Bennet, 2011). Para ello les ofrecemos que usen tantas tutorías como requieran, para que "hagan suyo" el material e interioricen los conocimientos, posibilitándoles el realizar preguntas incluso más allá de plan de estudios.

En éste caso concreto, la modalidad escogida ha sido multiplicar el tiempo dedicado a casos de seminario, manteniendo su peso en el total de la asignatura, para permitir un mayor trabajo en aula y fuera de ella sobre aspectos más prácticos y, además, posibilitando que los exámenes sean reemplazados por otra batería de casos, a desarrollar conforme van avanzando por la materia. Éstas tareas las han de realizar todos, dejando a su elección si se les calificarán o prefieren acceder al examen regular. Ésta opción, hay que reconocer que con sorpresa del docente, pues supone una carga adicional de trabajo para ellos, fue escogida por la totalidad de los alumnos. El objetivo perseguido era hacer más fácil la implicación del estudiante no solo con la materia, sino con el mecanismo docente empleado.

\section{Evaluaciones}

Las actividades y pruebas de evaluaciones ordinarias, en la modalidad no FLIP de la asignatura, pueden verse de forma esquemática en la tabla siguiente.En ella podemos apreciar que tipo de prueba es a la que se hace referencia, si ésta se desarrolla en una sesión de práctica, de teoría o de seminario de aula, cuantas y que forma toman, y el peso en la evaluación final. 
Su distribución a lo largo del tiempo pretende ser uniforme. Así, se reparten entre las sesiones de prácticas (excepto la primera y la última), los exámenes, dos, uno sobre el ecuador de la asignatura y otro al final de la misma, y los casos, cuatro, intentando que el tiempo transcurrido entre ellos sea equivalente. Para los exámenes convencionales y corrección de casos, se usa el sistema automático ALCE, disponible en poliformat. Por simplificar el modelo, obviamos los mecanismos de recuperación.

Tabla 1. Pruebas en la docencia ordinaria. Elaboración propia

\begin{tabular}{cccc}
\hline Tipo de prueba & $\begin{array}{c}\text { Corresponde } \\
\text { a }\end{array}$ & Prueba (número) & Valor en puntos \\
\hline $\begin{array}{c}\text { Examen } \\
\text { convencional, } \\
\text { papel, corrección } \\
\text { automática ALCE }\end{array}$ & Teoría aula & Examen parcial (2) & $3,5(1,5+2)$ \\
$\begin{array}{c}\text { Caso (apoyo con } \\
\text { documento pdf, } \\
\text { evaluación } \\
\text { automática ALCE) }\end{array}$ & Seminario & Debate + test (4) & \\
$\begin{array}{c}\text { Examen poliformat } \\
\text { Tarea Poliformat }\end{array}$ & Práctica & Test al final de la sesión (6) & $1,5(6 * 0,25)$ \\
& & Trabajo construido en grupo y & $0,5(0,25+0,25)$ \\
Tarea poliformat & Práctica & Trabajo individual & \\
\hline
\end{tabular}

$\mathrm{Al}$ establecer los cambios para la modalidad FLIP, se consideró, como ya se dijo, mantener los exámenes convencionales como una opción. Sin embargo, esa opción no fue escogida por nadie, por lo que para dar una mayor legiblilidad a la tabla siguiente, se excluye y aparecen tan solo aquellas pruebas usadas realmente.Como puede verse, las notas se descomponen en partes muy pequeñas. Eso implica que en el caso de no realizar, o fracasar en unas pocas, la continuidad en la asignatura no se resiente. Se intenta así evitar el abandono, al enganchar a los discentes en la sucesión, como un goteo, no molesto por su pequeño tamaño, pero persistentes. Los casos permiten "tocar la realidad", de forma que la aplicación de los conocimientos teóricos es inmediata. 
Diseño de una experiencia de Flip-Teaching para la asignatura Deontología y Profesionalismo a impartir en la Escuela Técnica Superior de Ingeniera Informática de la UPV

Para la evaluación de prácticas, al ser éstas realizadas en laboratorio, de forma idéntica a la modalidad clásica, el mecanismo no se ha alterado. Observemos que mientras los exámenes desaparecen (insistamos, por decisión de los propios alumnos), los casos se multiplican. Y, lo más importante, cambia el mecanismo de evaluación. Aquí desaparece ese micro examen, corregido con el mecanismo ALCE, que se empleaba en la versión tradicional. Para dar una mayor legiblidad, desglosaremos los casos por el tipo de herramienta empleada para su entrega y corrección. Conviene remarcar que los pesos de cada parte de la asignatura se han respetado, pero se ha ganado la posibilidad de poder distribuir mejor las evaluaciones atendiendo al peso de cada tema.

Tabla 2. Pruebas enmpleadas en modadlidad FLIP. Elaboración propia

\begin{tabular}{|c|c|c|c|}
\hline Tipo de prueba & $\begin{array}{l}\text { Corresponde } \\
\text { a }\end{array}$ & Prueba (número) & Valor en puntos \\
\hline $\begin{array}{l}\text { Caso (estudio en } \\
\text { seminario y } \\
\text { entrega de un } \\
\text { pequeño trabajo } \\
\text { relativo al mismo) }\end{array}$ & Seminario & $\begin{array}{l}\text { Recogida mediante tareas de } \\
\text { poliformat. Sustitución de } \\
\text { examen (2) }\end{array}$ & $1,5(0,75+0,75)$ \\
\hline $\begin{array}{l}\text { Caso (estudio en } \\
\text { seminario y } \\
\text { corrección } \\
\text { mediante examen } \\
\text { poliformat) }\end{array}$ & Seminario & $\begin{array}{l}\text { Debate + test (9) Seis de ellos, } \\
\text { correspondientes a los antiguos } \\
\text { casos, aligerados. Tres, como } \\
\text { sustitución de examen }\end{array}$ & 4,5 (0,5 c.u.) \\
\hline $\begin{array}{l}\text { Caso (estudio en } \\
\text { seminario y } \\
\text { evaluación en el } \\
\text { aula, presencial, de } \\
\text { forma oral) }\end{array}$ & Seminario & $\begin{array}{c}\text { Debate (3) Dos de ellos, similar a } \\
\text { los antiguos casos. Uno, como } \\
\text { sustitución de examen }\end{array}$ & 1,5 (0,5 c.u) \\
\hline Examen poliformat & Práctica & Test al final de la sesión (6) & $1,5(6 * 0,25)$ \\
\hline Tarea Poliformat & Práctica & $\begin{array}{l}\text { Trabajo construido en grupo y } \\
\text { coevaluación del mismo }\end{array}$ & $0,5(0,25+0,25)$ \\
\hline Tarea poliformat & Práctica & Trabajo individual & 0,5 \\
\hline
\end{tabular}




\section{Resultados}

Hay distintos elementos de medición aplicables a la docencia en general (Andrés, 2005) que resultan de interés por permitirnos comparativas entre grupos.

- $\quad$ Porcentaje de Asistencia del alumnado. Con éste indicador se alude al porcentaje de alumnos que no han faltado más del $20 \%$ de las horas lectivas. El dato viene condicionado por la indicación, en la guía docente de la asignatura, de una asistencia mínima a un $80 \%$ de la asignatura. El dato se expresa como la división entre el número de alumnos que cumplen la condición de asistencia y el número total de alumnos, multiplicado por cien. En nuestro caso la tasa es de un $100 \%$. Para el resto de alumnos, el dato es de un 90\%

- Porcentaje de aprobados: clásico indicador de rendimiento global, calculado como el número de alumnos con una nota igual o superior a 5, dividido por el total de matriculados, multiplicado por 100. En el caso de los alumnos que cursan por Flip teaching, al ir generando pequeñas calificaciones durante el curso, a pesar de que en el momento en que éstas líneas se escriben no ha finalizado, el dato es ya de un $100 \%$ de éxito. Ningún alumno ha suspendido la asignatura. Aun más, a falta de un par de pequeñas pruebas, la nota media puede rozar el notable. Para los alumnos no flip, pendientes del segundo parcial, de las últimas puntuaciones de prácticas y de un parcial, no es posible obtener una cifra comparativa. Como elemento significativo, con las notas actuales, de los alumnos que mantienen una asistencia regular, al menos un $13 \%$ de ellos van abocados al suspenso, aunque saquen una nota excelente en el último parcial de la asignatura. Dato que puede oscilar al alza cuando el resto de notas se publiquen, y moderado tras pruebas de recuperación (por ésta razón, es imposible dar una cifra comparativa de la tasa neta de aprobados, entendiendo como tal el rendimiento de los alumnos con la corrección necesaria para eliminar el posible fracaso del proceso de enseñanza aprendizaje por falta de esfuerzo o capacidad del alumnado, indicada mediante el cociente entre el número de alumnos aprobados y el de alumnos calificados positivamente que han asistido al $80 \%$ de las horas lectivas

- Porcentaje de contenidos impartidos, expresado como los contenidos impartidos, dividido por el total contenidos programados, multiplicado por cien. A falta de una semana de docencia, en todos los grupos de la asignatura (cinco convencionales, un grupo ARA, dos de retitulados y el grupo Flip) se va a cubrir la totalidad. La diferencia más palpable es que en el grupo FLIP un par de semanas antes, toda la teoría estaba ya considerada como impartida, dedicando el tiempo restante a actividades de seminario y prácticas. 
Diseño de una experiencia de Flip-Teaching para la asignatura Deontología y Profesionalismo a impartir en la Escuela Técnica Superior de Ingeniera Informática de la UPV

- Planificación de trabajos: porcentaje de trabajos entregados en plazo y conformes con las especificaciones. En el grupo FLIP, con una docena de actividades temporizadas en la plataforma, se advirtió en un par de ellas un ligero retraso, coincidente con la época en que los parciales de otras asignaturas cuajaban sus agendas. Este dato ha resultado significativo y ayudará a planificar mejor la agenda de entregas de próximas ediciones. Con respecto a la comparativa con los otros grupos, donde sólo hay dos actividades planificadas, una de ellas aun pendiente de entrega, no hay comparativa posible.

Al margen de éstos indicadores, hay otros elementos que pasan por la percepción del alumnado, y que sin dejar de ser muy interesantes para verificar el interés conseguido por la experiencia y el grado de aceptación de la materia en éste formato, son imposibles de captar sin su participación activa. Para éste fin, se ha diseñado un cuestionario, inspirado entre otros por (Bethany, 2012), para ser respondido por los alumnos al fin de la misma.

Los elementos que la componen oscilan en torno a puntos como:

- La utilidad de los contenidos, desde la percepción del alumnado

- La satisfacción de los alumnos tanto con los contenidos como con las herramientas empleadas y con el propio profesor

- Los tiempos invertidos realmente por ellos, como comparativo de los cálculos previos realizados en la planificación: número de horas dedicadas, carga de trabajo con respecto a otras materias...

- Observaciones que los alumnos quieran aportar al proceso

Podemos ver una imagen de la encuesta a continuación: 


\begin{tabular}{llllll}
\hline $\begin{array}{l}\text { Profesionalismo } \\
\text { informático }\end{array}$ & 0 & 0 & 0 & 0 & 0 \\
\hline $\begin{array}{l}\text { Marco legal } \\
\text { (introducción) }\end{array}$ & 0 & 0 & 0 & 0 & 0 \\
\hline $\begin{array}{l}\text { Protección de } \\
\text { datos }\end{array}$ & 0 & 0 & 0 & 0 & 0 \\
\hline $\begin{array}{l}\text { Propiedad } \\
\text { intelectual }\end{array}$ & 0 & 0 & 0 & 0 & 0 \\
\hline $\begin{array}{l}\text { Periciales } \\
\text { informáticas }\end{array}$ & 0 & 0 & 0 & 0 & 0 \\
\hline $\begin{array}{l}\text { Deontología } \\
\text { (introducción) }\end{array}$ & 0 & 0 & 0 & 0 & 0 \\
\hline $\begin{array}{l}\text { Deontología } \\
\text { informática }\end{array}$ & 0 & 0 & 0 & 0 & 0 \\
\hline
\end{tabular}

Sobre las herramientas empleadas... *

hemos usado distintas herramientas en poliformat, quisiera saber que te parecen

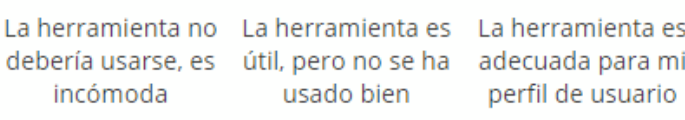

Debería usarse

más ésta

herramienta

\begin{tabular}{|c|c|c|c|c|}
\hline $\begin{array}{l}\text { Exámenes } \\
\text { poliformat (tests) }\end{array}$ & 0 & 0 & 0 & 0 \\
\hline $\begin{array}{l}\text { Entrega de tareas } \\
\text { mediante } \\
\text { poliformat }\end{array}$ & 0 & 0 & 0 & 0 \\
\hline $\begin{array}{l}\text { Foros de } \\
\text { discusión }\end{array}$ & 0 & 0 & 0 & 0 \\
\hline $\begin{array}{l}\text { Correo } \\
\text { electrónico (en y } \\
\text { fuera de } \\
\text { poliformat) }\end{array}$ & 0 & 0 & 0 & 0 \\
\hline
\end{tabular}

Sobre el profesor *

Fig. 2 Encuesta a los alumnos, fragmento.(Fuente: OLTRA)

Puede parecer temerario establecer conclusiones de una experiencia que aun no ha finalizado, sin embargo, tanto por un breve cuestionario realizado durante la primera semana como por los encuentros personales con los alumno y los resultados académicos que se van materializando, parecen confirmar que la experiencia no ha resultado precisamente un fracaso.

Obviamente, la educación superior en su formato pre-imprenta, donde solo el "sabio" posee el libro, está condenada a cambiar. La difusión multimedia, la universalización de la cultura que facilita Internet favorece la reconversión del docente en un guía, que es lo que se ha tratado de hacer aquí.

Evidentemente, al tratare de una experiencia con una extensión mínima de dos años, esto nos da pie a "jugar" con la misma, corrigiendo en el curso próximo los fallos que vamos

(cc) EY-NC-ND 2015, UniversitatPolitècnica de València

Congreso In-Red (2015) 
Diseño de una experiencia de Flip-Teaching para la asignatura Deontología y Profesionalismo a impartir en la Escuela Técnica Superior de Ingeniera Informática de la UPV

observando en el presente, como ese colapso temporal que en algún momento del curso parecen sufrir los alumnos por la coincidencia de trabajos y exámenes de múltiples asignaturas, como por el sobreesfuerzo del profesor a la hora de corregir. Una previsión de futuro es introducir más correcciones automáticas y autocorrecciones, que hagan más liviano el trabajo del docente.

Por otra parte, qué duda cabe, se facilita la reutilización de contenidos. Tanto para asignaturas de corte similar, en otras titulaciones, como para alumnos que no cursan la asignatura en ésta modalidad, pero que puede servirles de refuerzo el disponer de una alternativa a los apuntes clásicos para su estudio.

Las perspectivas son, pues, optimistas.

\section{Referencias}

ANDRÉS, M.A. (2005). "Propuesta de indicadores del proceso de enseñanza/aprendizaje en la formación profesional en un contexto de gestión de calidad total" en Revista ELectrónica de Investigación y EValuación Educativa, v. 11, n.1

<http://www.uv.es/RELIEVE/v10n2/RELIEVEv11n1_4.htm> [Consulta: 2 de mayo 2015]

BENNETT B., KERN J., GUDENRATH A., MCINTOSH P (2011). "The Flipped Class Revealed" en The Daily Riff. < http://www.thedailyriff.com/articles/the-flipped-class-what-does-a-goodone-look-like-692.php> [Consulta: 15 de mayo de 2015]

BETHANY B. S- (2012) "Flip Your Classroom to Increase Active Learning and Student Engagement"VV.AA. En 28th Annual Conference on Distance Teaching \& Learning Wisconsin, EE.UU. University of Wisconsin, 1-5

BLOOM, B. S. (1981) Taxonomia de los objetivos de la educación. Buenos Aires,Libreria de Ateneo

DOMINGO, J. (2015) Técnicas de trabajo en Aprendizaje basado en problemas/proyectos PBL.Valencia, UPV-ICE

MOWAFY A., KUHN, M., SNOW, T (2013) "Blended learning in higher education: Current and future challenges in surveying education in Issues" en Educational Research, 23(2): Special Issue, 132-150

OLTRA, J. Encuesta DYP-FLIP 2015<http://xurl.es/dypflip2015> [Consulta: 25 de mayo 2015]

ROSHAN S. (2011) "The best way to reach each student? Private school Math teacher flips learning" en The Daily Riff. < http://www.thedailyriff.com/articles/the-best-way-to-reach-each-studentprivate-school-flips-learning-547.php> [Consulta: 2 de mayo 2015]

SAMS A., BERGMANN J. (2013) "Flip Your Students' Learning" en Technology-Rich Learning Vol $70 \mathrm{n}^{\circ} 6$

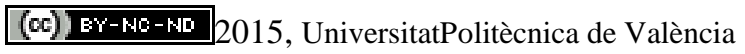

Congreso IN-RED (2015) 
SPENCER D., WOLF D.\& SAMS A (2011) "Are you ready to flip? " En The Daily

Riff. $<$ http://www.thedailyriff.com/articles/are-you-ready-to-flip-691.php> [Consulta: 2 de mayo 2015]

VASILEVA-STOJANOVSKA T., MALINOVSKI T., DOBRIJOVEVSKI M.V., TRAJKOVIK V. (2015) "Impact of satisfaction, personality and learning style on educational outcomes in a blended learning environment" En Learning and Individual Differences (Article in press) $<$ do:10.1016/j.lindif.2015.01.018> [Consulta: 18 de mayo 2015] 